\title{
Mast cell activation syndromes - evaluation of current diagnostic criteria and laboratory tools in clinical practice (Review)
}

\author{
POLLIANA MIHAELA LERU ${ }^{1,2}$, VLAD FLORIN ANTON $^{2}$, CORINA URECHE $^{3}$, SABINA ZURAC $^{1,4}$, \\ OVIDIU BRATU ${ }^{1,5,6}$ and CARMEN DANIELA NEAGOE ${ }^{7,8}$ \\ ${ }^{1}$ Family Medicine Department, 'Carol Davila' University of Medicine and Pharmacy, 050474 Bucharest; \\ ${ }^{2}$ Internal Medicine Clinic, 'Colentina' Clinical Hospital, 020125 Bucharest; ${ }^{3}$ Internal Medicine Clinic, \\ 'George Emil Palade' University of Medicine, Pharmacy, Science and Technology, 540139 Târgu Mureș; \\ ${ }^{4}$ Department of Pathology, 'Colentina' Clinical Hospital, 020125 Bucharest; ${ }^{5}$ Urology Clinic, \\ Central Military Emergency University Hospital, 010825 Bucharest; ${ }^{6}$ Academy of Romanian Scientists, \\ 050045 Bucharest; ${ }^{7}$ Internal Medicine and Gastroenterology Clinic, Emergency County Hospital, 200642 Craiova; \\ ${ }^{8}$ Gastroenterology Department, University of Medicine and Pharmacy, 200349 Craiova, Romania
}

Received May 15, 2020; Accepted June 16, 2020

DOI: $10.3892 /$ etm.2020.8947

\begin{abstract}
Mast cell activation syndromes (MCAS) represent a heterogeneous clinical entity caused by episodic and severe mast cell activation, including primary and secondary mast cell disorders (MCDs). The group of primary or clonal MCDs refers to systemic mastocytosis, other clonal MCAS and hereditary hypertryptasemia, while the secondary MCAS is characterized by normal mast cells (MCs) which are activated by external triggers, such as allergens or physical stimuli. Another category of MCAS is the idiopathic form, when no trigger or genetic mutation can be identified. Symptoms of mast cell activation are due to release of specific mediators and can be seen in many diseases, such as allergies, with localized or systemic clinical manifestations. Confirmation of MCAS is based on diagnostic criteria proposed by an international group of experts and the best available evidence in this field. It is generally accepted that the clinical picture of MCAS is non-specific and there are few available laboratory tools, making it difficult for clinicians to identify and confirm this entity. The diagnosis is established after exclusion of other possible clinical entities in most of the cases. Therefore, the actual diagnostic criteria of MCASs, some relevant clinical aspects and laboratory tools used in clinical practice were reviewed.
\end{abstract}

Correspondence to: Dr Polliana Mihaela Leru, Internal Medicine Clinic, 'Colentina' Clinical Hospital, 19-21 Sos. Stefan cel Mare, 020125 Bucharest, Romania

E-mail: polianaleru@yahoo.com

Key words: anaphylaxis, mast cells, mast cell activation syndromes, rare diseases, tryptase

\section{Contents}

1. Introduction

2. Classification and clinical forms of mast cell activation syndromes and disorders

3. Consensus diagnostic criteria for MCAS

4. Mast cell mediators and their clinical use in diagnosing MCAS

5. Differential diagnosis of MCAS

6. Discussion

\section{Introduction}

Mast cells (MCs) were discovered in 1879 by Paul Ehrlich, who is considered the founder of modern immunology and they have been long considered to play mostly proinflammatory roles in allergic reactions (1). The scientific interest for MCs has very much increased during the last decades and recent research was able to prove their more complex implication in human pathology, such as cancer, infections, cardiovascular diseases and various systemic disorders (2).

MCs play important immunomodulatory roles, releasing a broad range of proinflammatory as well as anti-inflammatory mediators influencing the interaction of the immune system with the environment. Their predominant location in the skin, the gastrointestinal tract and the respiratory system permit the MCs to react as a first responder to any change in the environment. It was proven that MCs may react to various stimuli and intervene in pathologic processes even if they are not the central pathogenic player (3). In different conditions, MC dysfunction or abnormal location, with release of specific mediators, may result in a broad range of MCs activation disorders (MCDs) (4). There are no accurate epidemiological data regarding MCDs. The prevalence of mastocytosis is estimated at 2.6 in 10,000 people in European Union (www.ema.europa.eu) and an annual global incidence of 5-10 new cases per million population was reported (5). No data on MCAS prevalence could be found. 


\section{Classification and clinical forms of mast cell activation syndromes and disorders}

Mast cell activation syndromes (MCAS) represent a heterogeneous group of disorders, ranging from very rare to very common, caused by episodic and severe spontaneous activation and degranulation of MCs. Abnormal activation of MCs can result from two distinct pathological conditions. The first and rare condition is due to an excessive production of abnormal MCs, such as the case of clonal mast cell disorder-mastocytosis. The neoplastic MCs proliferate due to a gain-of-function mutation, mostly in KIT, a transmembrane receptor tyrosine kinase, expressed by the MCs. The abnormal activated MCs infiltrate tissues and release their mediators, resulting in a broad range of clinical signs and symptoms. The second and common condition is the exaggerate activation of MCs, as a defense over-reaction against a perceived threat, such is the case of allergic reactions, infections or physical triggers (6).

Primary MCDs include mastocytosis and monoclonal mast cell activation syndrome (MMCAS), a spectrum of rare and well-defined diseases associated with clonal expansion of MCs in the skin and/or other tissues and organs, most commonly the bone marrow, gastrointestinal tract, liver, spleen and lymph nodes. Other rare diseases in this group are mast cell sarcoma, mast cell leukemia and hereditary hypertryptasemia (7).

Clinical presentation of systemic mastocytosis (SM) in adults is very heterogeneous and the diagnosis can be more challenging in the absence of skin involvement, due to variable and multiple organ dysfunction. Patients with cutaneous and/or systemic mastocytosis may suffer symptoms due to MC activation and release of mast cell mediators, mostly after exposure to physical, psychological, medication or food triggers (8).

Positive diagnosis of SM requires the presence of the major criterion, multifocal MC infiltration in the bone marrow and/or other extracutaneous organ plus one minor criterion or at least three of the four minor criteria: Abnormal MC expression of $\mathrm{CD} 25$ and/or CD2, $>25 \%$ atypical $\mathrm{MC}$ in the bone marrow infiltrates, evidence of the characteristic c-KIT mutation and increased serum tryptase $>20 \mathrm{ng} / \mathrm{ml}$ (9).

Two particular forms of systemic mastocytosis include adult patients with anaphylaxis induced by hymenoptera venom, with no skin lesions and also childhood cutaneous mastocytosis progressing to adult systemic disease, despite missing the characteristic gene mutation (10).

Diseases due to secondary mast cell activation include hypersensitivity reactions, caused by both $\mathrm{IgE}$-mediated and non IgE-mediated triggers, but also physical urticarias, some chronic infections and systemic diseases with mast cell hyperplasia and activation (6). Abnormal MC activation can be localized, limited to one organ, such is the case of rhinitis, wheezing and urticaria or systemic, with anaphylaxis as the extreme example (11).

Regarding diagnosis of mast cell activation syndrome (MCAS), it must be noted that this is mostly a clinical one, with clinical suspicion raised based on patient history and after exclusion of other possible disorders. The confirmation of MCAS might be difficult in clinical practice, also due to few available laboratory tests. Experts recommend that it should be considered in patients with appropriate clinical and laboratory profile, when all consensus criteria are fulfilled and other conditions have been excluded (12).

\section{Consensus diagnostic criteria for MCAS}

i) Episodic and recurrent symptoms of mast cell mediator release, affecting two or more organ systems (often in the form of anaphylaxis). ii) Complete resolution of symptoms or decrease in the frequency or severity of symptoms with anti-mast cell mediator therapy (antihistamines, leukotrienes modifiers and mast cell stabilizer agents). iii) Evidence of increase in a validated urinary or serum marker of MCAS (preferably with reproducible results obtained during more than one symptomatic episode) (12).

There is no single pathognomonic clinical presentation for MCAS, therefore all three consensus criteria should be fulfilled in order to confirm the diagnosis (13).

Patients with MCAS may have variable clinical phenotype, affecting multiple organ systems, but the key feature is recurrent episodes of severe symptoms (anaphylaxis), with concurrent involvement of minimum two organ systems and association with acute increase of specific biologic mediator levels. Idiopathic anaphylaxis is considered a subtype of MCAS, since MCs are the only effector cells of anaphylaxis in humans, when no possible cause or stimulus can be proven (7). Persistent symptoms, such as chronic urticaria or uncontrolled asthma, as well as chronic increases of biologic mediators do not support MCAS diagnosis. The most frequently symptoms reported by patients with MCAS, due to MCs mediator release are: cardiovascularhypotension, tachycardia, syncope; cutaneous-pruritus, urticaria, flushing, angioedema; respiratory-wheezing, stridor; gastrointestinal-crampy abdominal pain, diarrhea, nausea and vomiting (14).

\section{Mast cell mediators and their clinical use in diagnosing MCAS}

MCAS symptoms are due to secretion of MC products, such as histamine, tryptase, prostaglandin D2 and leukotrienes, with few of them clinically useful as markers of MCs activation and validated laboratory tools for diagnostic confirmation (15) (Table I).

Serum tryptase is the most specific marker of MC activation and is currently used as a reliable diagnostic tool for confirmation of MCDs, including MCAS, providing its correct measurement and interpretation (16). The serum tryptase level usually increases during mast cell degranulation, with a peak between 1 and $4 \mathrm{~h}$. A normal tryptase level is considered $5 \mathrm{ng} / \mathrm{ml}$, a level greater than $11.4 \mathrm{ng} / \mathrm{ml}$ is considered elevated and a level greater than $20 \mathrm{ng} / \mathrm{ml}$ is a minor diagnostic criterion for systemic mastocytosis (17). According to consensus criteria for MCAS diagnosis, a change of $20 \%$ of baseline serum tryptase level plus $2 \mathrm{ng} / \mathrm{ml}$ is indicative for a mast cell activation episode, mostly due to systemic immediate hypersensitivity reactions or anaphylaxis (12).

\section{Differential diagnosis of MCAS}

Many diseases can be considered as alternate possible diagnostic when evaluating MCAS in clinical practice (Table II).

A subgroup of patients with MCDs may associate concomitant blood or tissue hypereosinophilia (HE), possibly due to shared roles of MCs and eosinophils in many diseases, classically associated with the pathogenesis of allergic diseases $(18,19)$. MCs 
Table I. Clinically available and validated markers of mast cell activation (adapted from Akin, 2017) (6).

\begin{tabular}{ll}
\hline Marker & Diagnostic value and clinical interpretation \\
\hline Serum tryptase & $\begin{array}{l}\text { The most specific marker, almost always increased in patients with severe mast cell } \\
\text { activation episodes (anaphylaxis). } \\
\text { Repeated measurements are needed: within } 4 \mathrm{~h} \text { of an episode and compared } \\
\text { with baseline values (outside of MCs activation). }\end{array}$ \\
$\begin{array}{l}\text { Increased baseline levels might indicate mastocytosis or familial hypertryptasemia } \\
\text { (after excluding renal disease or myeloid neoplasm). }\end{array}$ \\
$\begin{array}{l}\text { Fairly specific for mast cell activation, may be influenced by diet or bacterial } \\
\text { contamination. } \\
\text { Specific cutoffs for MCAS is not established. }\end{array}$ \\
Urinary histamine metabolites & $\begin{array}{l}\text { Increased in patients with mast cell activation, but not recommended as the single } \\
\text { marker of mast cell activation. }\end{array}$ \\
Can guide the decision to initiate aspirin therapy (except the patients with allergy \\
to nonsteroidal anti-inflammatory drugs). \\
Increased in patients with mast cell activation, but less clinical value and \\
experience comparing to other markers. \\
Might guide the decision to initiate leukotriene-targeting therapy.
\end{tabular}

MCs, mast cells; MCAS, mast cell activation syndromes.

Table II. Diseases currently considered for differential diagnosis of MCAS (adapted from Khokhar and Akin, 2020) (7).

\begin{abstract}
Vasovagal syncope and postural orthostatic tachycardia syndrome
Endocrine: Carcinoid syndrome, pheochromocytoma, thyroid cancer, Addison disease

Cutaneous: Chronic urticaria, dermatomyositis, idiopathic flushing, contact or atopic dermatitis

Neuropsychiatric: Panic attacks, epilepsy, multiple sclerosis, eating disorders

Hereditary hypertryptasemia

Hereditary or acquired angioedema

Gastrointestinal: Irritable bowel syndrome, inflammatory bowel disease, ulcer disease, eosinophilic gastrointestinal disorders

Cardiac: Arrythmias, coronary artery disease

Drug adverse effects: Niacin-induced flushing, steroid toxicity, withdrawal of adrenergic medications, sympathomimetics, anticholinergic toxicity, caffeine effect, alcohol use or withdrawal
\end{abstract}

MCAS, mast cell activation syndromes.

role in parasitic infections is much less clear compared with that of eosinophils, despite considering infections as possible triggers for activation of MCs in some individuals. Recent data from literature confirmed the bidirectional interactions between these cells and their important implication not only in allergic diseases, but also in many inflammatory, cardiovascular and neoplastic disorders (20). An evaluation of the prognostic impact of eosinophils in mastocytosis patients, concluded that HE did not influence mediator-related symptoms or allergic reactions, but was related with worse prognosis, compared with mastocytosis patients without eosinophilia (21). Since both MCDs and eosinophilic diseases are rare and sometimes poorly understood, their association brings even more difficulty in adequately diagnosing and treating these complex pathological conditions.

\section{Discussion}

MCDs, including MCAS and systemic mastocytosis, are rare diseases, generally addressed to different medical specialists, depending on the clinical presentation and the most prominent symptoms. Long delay until diagnosis confirmation is often reported, due to rather low awareness of the disease within different medical specialties. A high index of suspicion is required mainly in the clinical setting of unexplained anaphylaxis, osteoporosis in young patients, episodes of flushing, persistent and refractory abdominal cramping or gastrointestinal disease. Many patients may ask allergist evaluation for presumptive intolerances or multiple food or drug allergies that need complex evaluation (22). In other patients, more severe or refractory cutaneous diseases or symptoms aggravated by psychological triggers may indicate possible rare diseases, including MCAS $(23,24)$. Given the continuous increasing prevalence of allergic diseases worldwide, the problematic allergies or possible allergic reactions became stringent, also related to MCAS and diseases. There are still difficulties and differences between countries in practicing allergology specialty, with rather low number of specialists, unrecognized cases and high costs for laboratory tests, as have been showed by a local study (25). 
The diagnosis and management of MCDs is difficult in clinical practice, requiring vast knowledge, multidisciplinary approach and personalized medicine procedures (26). Many patients may consider themselves as having MCAS, due to a variety of persistent multisystem symptoms, including chronic fatigue, intolerances to various environmental factors, food and medication, memory loss or headache, with no clear cause, despite complex medical evaluation. The is no scientific evidence suggesting that an abnormal mast cell phenotype could be responsible for this type of chronic clinical pattern and a high index of suspicion is very important before addressing patients with possible MCDs to allergists or other specialists.

In conclusion, MCAS represent a rare and difficult to manage clinical entity. Diagnosis confirmation may take a long time, involve unpleasant diagnostic procedures and high costs, leading to frustration of both patients and doctors. There is a clear need for developing research and multidisciplinary collaboration in the field of allergic and rare diseases, including mast cell disorders, in order to improve diagnosis and management of this complex pathology.

\section{Acknowledgements}

Not applicable.

\section{Funding}

No funding was received.

\section{Availability of data and materials}

Not applicable.

\section{Authors' contributions}

PML designed the study and wrote the manuscript. VFA, CU, SZ, OB and CDN contributed to the drafting of the manuscript and were involved in the conception of the study. All authors read and approved the final manuscript.

\section{Ethics approval and consent to participate}

Not applicable.

\section{Patient consent for publication}

Not applicable.

\section{Competing interests}

The authors declare that they have no competing interests.

\section{References}

1. Ehrlich P: Contributions to the Theory and Practice of Histological staining. PhD Thesis. University of Leibzig, Leibzig, 1878.

2. Varricchi G and Marone G: Mast cells: Fascinating but still elusive after 140 years from their discovery. Int J Mol Sci 21: 464, 2020.
3. Theoharides TC, Valent P and Akin C: Mast cells, mastocytosis and related disorders. N Engl J Med 373: 163-172, 2015.

4. Metcalfe DD and Akin C: Mastocytosis: Molecular mechanisms and clinical disease heterogeneity. Leuk Res 25: 577-582, 2001.

5. Hartmann K and Henz BM: Mastocytosis: Recent advances in defining the disease. Br J Dermatol 144: 682-695, 2001.

6. Akin C: Mast cell activation syndromes. J Allergy Clin Immunol 140: 349-355, 2017.

7. Khokhar D and Akin C: Mast cell activation. When the whole is greater than the sum of its parts. Med Clin North Am 104: 177-187, 2020.

8. Valent P, Akin C, Gleixner KV, Sperr WR, Reiter A, Arock M and Triggiani M: Multidisciplinary challenges in mastocytosis and how to address with personalized medicine approaches. Int J Mol Sci 20: 2976, 2019.

9. Pardanani A; Annual Clinical Updates in Hematological Malignancies: Systemic mastocytosis in adults: 2019 update on diagnosis, risk stratification and management. Am J Hematol 94: 363-377, 2019

10. Bonadonna P, Zanotti R, Pagani M, Caruso B, Perbellini O, Colarossi S, Olivieri E, Dama A, Schiappoli M, Senna G, et al: How much specific is the association between hymenoptera venom allergy and mastocytosis? Allergy 64: 1379-1382, 2009.

11. Nilsson G and Dahlin JS: New insights into the origin of mast cells. Allergy 74: 844-845, 2019.

12. Weiler CR, Austen KF, Akin C, Barkoff MS, Bernstein JA, Bonadonna P, Butterfield JH, Carter M, Fox CC, Maitland A, et al: AAAAI Mast Cell Disorders Committee Work Group Report: Mast cell activation syndrome (MCAS) diagnosis and management. J Allergy Clin Immunol 144: 883-896, 2019.

13. Valent P, Akin C, Bonadonna P, Hartmann K, Brockow K, Niedoszytko M, Nedoszytko B, Siebenhaar F, Sperr WR, Oude Elberink JN, et al: Proposed diagnostic algorithm for patients with suspected mast cell activation syndrome. J Allergy Clin Immunol Pract 7: 1125-1133.e1, 2019.

14. Picard M, Giavina-Bianchi P, Mezzano V and Castells M: Expanding spectrum of mast cell activation disorders: Monoclonal and idiopathic mast cell activation syndromes. Clin Ther 35: 548-562, 2013.

15. Khoury P and Lyons JJ: Mast cell activation in the context of elevated basal serum tryptase: Genetics and presentations. Curr Allergy Asthma Rep 19: 55, 2019.

16. Schwartz LB: Diagnostic value of tryptase in anaphylaxis and mastocytosis. Immunol Allergy Clin North Am 26: 451-463, 2006.

17. Ahmed F, Fried AJ, Spielman S, Secor W and MacGinnitie AJ: Utility of measuring tryptase levels in children and young adults. Ann Allergy Asthma Immunol 123: 398-399, 2019.

18. Leru PM: Eosinophilia and hypereosinophilic disorders - update on etiopathogeny, classification and clinical approach. Rom J Intern Med 53: 289-295, 2015.

19. Leru PM: Eosinophilic disorders: Evaluation of current classification and diagnostic criteria, proposal of a practical diagnostic algorithm. Clin Transl Allergy 9: 36, 2019.

20. Galdiero MR, Varricchi G, Seaf M, Marone G, Levi-Schaffer F and Marone G: Bidirectional mast cell-eosinophil interactions in inflammatory disorders and cancer. Front Med (Lausanne) 4: $103,2017$.

21. Kluin-Nelemans HC, Reiter A, Illerhaus A, van Anrooij B, Hartmann K, Span LF, Gorska A, Niedoszytko M, Lange M, et al: Prognostic impact of eosinophils in mastocytosis: Analysis of 2350 patients collected in the ECNM Registry. Leukemia 34: 1090-1101, 2019.

22. Manea I, Ailenei E, Candrea E, Dima D and Deleanu D: Diagnostic challenge in a patient with severe pruritic rash. Ann Allergy Asthma Immunol 114: 65-67, 2015.

23. Grigore O, Mihailescu AI, Solomon I, Boda D and Caruntu C: Role of stress in modulation of skin neurogenic inflammation. Exp Ther Med 17: 997-1003, 2019.

24. Solomon I, Ilie MA, Draghici C, Voiculescu VM, Căruntu C, Boda D and Zurac S: The impact of lifestyle factors on evolution of atopic dermatitis: An alternative approach. Exp Ther Med 17: 1078-1084, 2019.

25. Leru PM and Deleanu DM: Romanian allergology in the actual European context. Rom J Intern Med 53: 111-117, 2015.

26. Castells M and Butterfield J: Mast cell activation syndrome and mastocytosis: Initial treatment options and long-term management. J Allergy Clin Immunol Pract 7: 1097-1106, 2019. 